\title{
Quantification and classification of microstructures in ternary eutectic alloys using 2-Point spatial correlations and principal component analyses
}

\author{
Abhik Choudhury $^{\mathrm{a}}$, Yuksel C. Yabansu ${ }^{\mathrm{b}}$, Surya R. Kalidindi ${ }^{\mathrm{b}, \mathrm{c}}$, Anne Dennstedt ${ }^{\mathrm{d}}$ \\ ${ }^{a}$ Department of Materials Engineering, Indian Institute of Science, 560012 Bangalore, India \\ ${ }^{b}$ George W. Woodruff School of Mechanical Engineering, Atlanta, GA 30332, United States \\ ${ }^{c}$ School of Computational Science and Engineering, Georgia Institute of Technology, Atlanta 30332, GA, USA \\ ${ }^{d}$ Institut für Materialphysik im Weltraum, Deutsches Zentrum für Luft- und Raumfahrt (DLR), 51170 Köln, Germany
}

\begin{abstract}
Eutectic solidification gives rise to a number of distinct microstructure patterns that might include lamella, rods and labyrinths in binary alloys. However, as the number of phases and components increases, the number of possible patterns that might be obtained during bulk solidification also become larger. While the morphological attributes of binary eutectic solidification have been fairly well understood, the same is not true for ternary and higher multicomponent alloys. In this paper, we study and quantify microstructures in ternary alloys as a function of two essential parameters, namely, the volume fraction of the solid phases and the surface energies of the interfaces (in particular the solid-liquid interfaces). For the selected ensemble of microstructures, quantification and classification were carried out using a recently developed data-driven (objective) approach based on principal component analyses of 2-point correlations. It is demonstrated that the method is capable of analyzing and quantifying the similarity/difference measures between the elements of the selected ensemble of microstructures.
\end{abstract}

Keywords:

Ternary Eutectic, Phase-Field, CALPHAD, pattern-formation, PCA

\section{Introduction}

Three-phase, ternary eutectic growth has been a topic of high interest for both experimentalists as well as theoreticians owing to the rich variety of patterns or microstructures possible. Rigorous study of these microstructures can provide an improved understanding of the principle mechanisms of general importance for the development of multicomponent alloys needed in advanced technology applications. Experimental investigations of ternary alloys have been carried out for both metallic [1] and inorganic alloys [2] for thin-film solidification conditions. Bulk solidification of three-phase growth has also been studied extensively [3-9]. However, theoretical investigations of threephase growth have been few. Himemiya and Umeda [10] have worked out analytical expressions for undercooling as a function of spacings for different three-phase configurations. Modeling efforts for thin-film and bulk solidification patterns were described in a few papers $[11,12]$.

One of the widely studied alloys in this regard is the Ag-Al-Cu alloy [13-18] which classically shows patterns of the type as shown in Fig. 1(a). However, there are

Email addresses: abhiknc@materials.iisc.ernet.in (Abhik Choudhury), yabansu@gatech.edu (Yuksel C. Yabansu), surya.kalidindi@me.gatech.edu (Surya R. Kalidindi), anne.dennstedt@gmx.com (Anne Dennstedt) other systems such as the Nb-Al-Ni ternary eutectic system [19] that exhibit quite different patterns compared to those seen in the Ag-Al-Cu alloy. These observations raise interesting questions regarding the correlation of microstructure features to material and/or processing parameters.

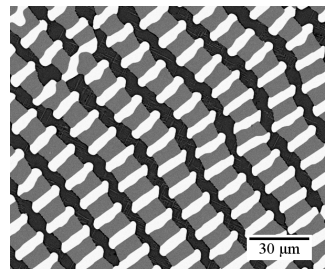

(a)

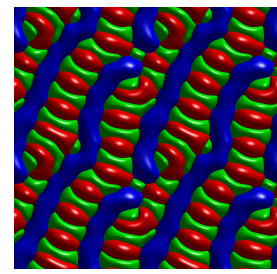

(b)
Figure 1: In (a) a typical microstructures in a directionally solidified $\mathrm{Ag}-\mathrm{Al}-\mathrm{Cu}$ ternary eutectic alloy [20], white: $\mathrm{Ag}_{2} \mathrm{Al}$, grey: $\mathrm{Al}_{2} \mathrm{Cu}$, black: $A l$. In (b) a phase-field simulation of the same system showing similar morphological characteristics as in the experiment, but with different ordering of the phases. Blue: $\mathrm{Al}_{2} \mathrm{Cu}$, green: $\mathrm{Ag}_{2} \mathrm{Al}$, red: $A l$.

In recent work [21], a phase-field simulation study was performed to understand how surface energies and volume fractions influenced the microstructure pattern formation during three dimensional directional solidification. For this purpose, the authors started with a symmetric model 
ternary eutectic alloy with equal surface energies, and investigated the individual influence of volume fractions and surface energies. For the study on the influence of volume fractions, continuous simulations were performed along the two composition pathways shown in Fig.2. In this set of simulations, the composition of the far-field liquid was changed periodically after sufficient time-intervals, during which the pattern at a given composition of the liquid is allowed to get reasonably close to the steady-state condition.

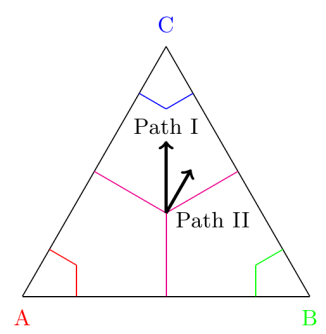

Figure 2: Schematic of the liqiudus projections (magenta) and solidus projections of the three solid phases, along with the two composition pathways for which the simulations were performed.

Thereafter, two simulations were performed to investigate the influence of solid-liquid interfacial energies both independently and in combination with change in volume fractions. Additionally, some insights into pattern formation of real alloys were also derived from phase-field simulations coupled with thermodynamic databases. From these, it was possible to derive structures displayed in Fig.1(b), which resemble the experimental structures highlighted in Fig.1(a).

While, the simulation study was able to span a wide range of volume fractions, solid-liquid surface energies and also a different phase diagram, the study remains qualitative in terms of quantifying the different structures obtained. Also, there is a critical need to extend the study to include different combinations of the solid-solid surface energies.

In this work, we draw upon the simulation results from the previous work referenced above [21], and add critical missing microstructures; this completes the ensemble of microstructures depicting the wide variety of possibilities of structure formation in three-phase systems. Thereafter, we present an analysis of some of the geometrical features that exhibit the highest sensitivity to changes in the volume fractions. As we will see, a change in the processing conditions or the processing parameters brings upon changes in multiple features in the microstructure. As a result, it often becomes difficult to identify or extract a precise correlation between the process parameters and the predicted microstructure. In this paper, we have therefore decided to explore these correlations using a much more objective and quantitative framework. This recently formulated framework employs two-point spatial correla- tions to rigorously quantify the material microstructure in a statistical framework. The method subsequently employs principal component analysis (PCA) to obtain an objective low-dimensional representation of the material microstructure. Since PCA accomplishes the dimensionality reduction in ways that maximizes the capture of the variance between the elements of the dataset in the minimum number of terms, it is ideally suited for our purpose. The PC representations are then used to quantify the magnitude of resemblance/differences between the different microstructures obtained in the simulation study.

\section{Brief summary of the simulation results}

\subsection{Microstructures from prior work}

In this section, we recall the simulation results from Ref. [21], as they will be included in the statistical analysis conducted in this study. For the purely symmetric ternary eutectic phase-diagram, with equal surface energies for all interfaces, the phase-field simulations give rise to a mostly regular polygonal structure of the form highlighted in Fig. 3.

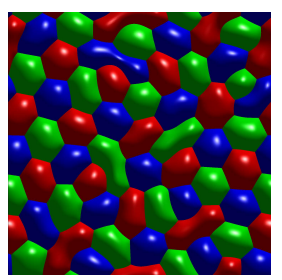

Figure 3: Phase-field simulation of purely symmetric ternary eutectic system at the eutectic composition.

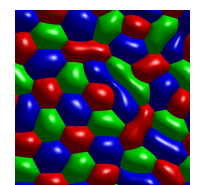

(a)

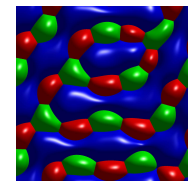

(d)

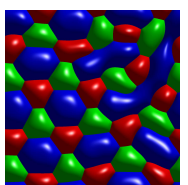

(b)

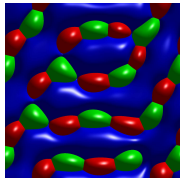

(e)

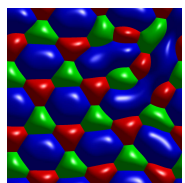

(c)

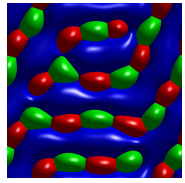

(f)
Figure 4: Series of microstructures that are obtained upon changing the volume fractions along a composition pathway along which volume fractions of two phases (red and green) remain equal (Path I). The volume fractions corresponding to the images are listed in order for (red, green, blue) phases as (a): $(0.31,0.31,0.38)$, (b): $(0.26,0.26,0.48), \quad(\mathrm{c}):(0.24,0.24,0.52), \quad(\mathrm{d}):(0.22,0.22,0.56), \quad(\mathrm{e}):$ $(0.2,0.2,0.6),(\mathrm{f}):(0.2,0.2,0.6)([21])$.

Fig. 4 and Fig. 5 represent simulated microstructures along Path I and Path II, respectively, as described in Fig.2 


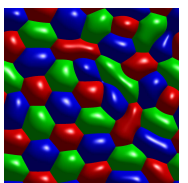

(a)

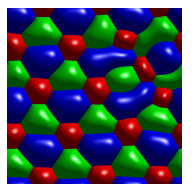

(d)

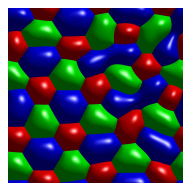

(b)

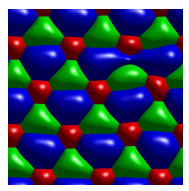

(e)

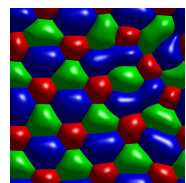

(c)

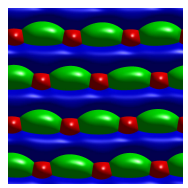

(f)
Figure 5: Series of microstructures that are obtained upon changing the volume fractions along a composition pathway, wherein the volume fractions of one of the phases (green) remains constant (Path II). The volume fractions corresponding to the images are listed in order for (red, green, blue) phases as (a): $(0.31,0.33,0.36)$, (b): $(0.26,0.33,0.41), \quad(\mathrm{c}):(0.25,0.33,0.42), \quad(\mathrm{d}):(0.21,0.33,0.46), \quad$ (e): $(0.19,0.33,0.48)$, (f): $(0.12,0.33,0.55)([21])$.

(cf. [21]). An interesting question one could ask is: what would happen, if we were to take back the composition to the ternary eutectic point. To address this, we directly ramped up the far-field composition from the final state in Fig. 5(f), and were able to get to the complete hexagonal state, with a regular-brick structure in the intermediate evolution, Fig.6.

In order to access the influence of surface energies we performed calculations wherein, the solid-liquid interfacial energies of one of the phases (red phase in Fig. 7(a)) is lowered for a completely symmetric phase diagram. A second calculation is also performed where the solid-liquid interfacial energy of one of the phases is lowered along with an increase in its equilibrium volume fraction at the eutectic temperature. The results are highlighted in Fig. 7(b). More details can be found in Ref. [21].

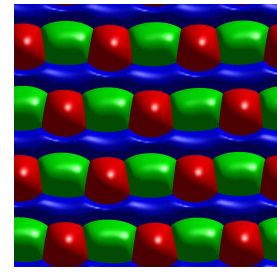

(a)

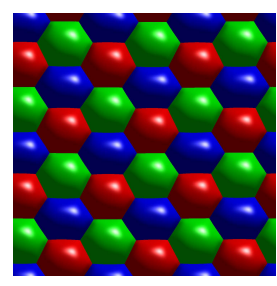

(b)
Figure 6: Revamping the composition towards the eutectic composition leads to the formation of the purely hexagonal state in the end (b), as would be expected out of the symmetry of the material parameters. The evolution follows through the breakdown of the brick structure, an intermediate stage being highlighted in (a).

In prior work, we also performed simulations of the $\mathrm{Ag}-\mathrm{Al}-\mathrm{Cu}$ alloy, where we linked the phase-field model to thermodynamic databases. Details about the thermodynamic coupling, surface energies, non-dimensionalization and simulation conditions can be found in detail in [21].

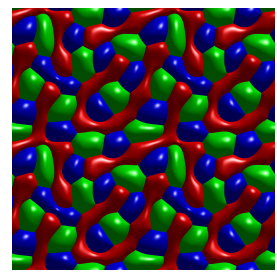

(a)

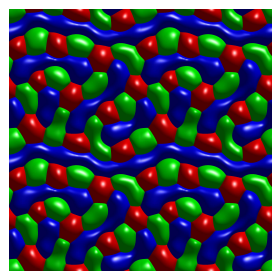

(b)
Figure 7: Effect of the influence of the surface energy on the predicted microstructures. In (a) phase-fractions corresponding to the ternary eutectic composition in the equilibrium phase diagram are equal, while one of the phases (red) has a solid-liquid interfacial energy lower than the other phases. In (b) the equilibrium volume fractions of two of the phases (red and green) is smaller than the blue phase, which is also the least stiff. Both figures are tiled $2 \times 2$ for better visualization.

The results from these phase-field simulations of the Ag$\mathrm{Al}-\mathrm{Cu}$ alloy are highlighted in Fig. 8, and are included in the present study.

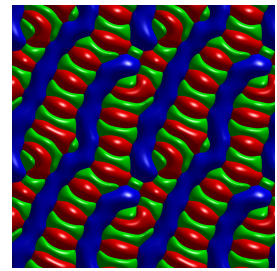

(a)

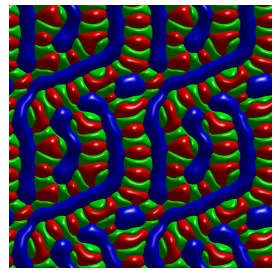

(b)
Figure 8: Simulations of the Ag-Al-Cu alloy at two different temperatures (a) $T=0.975$ and (b) $T=0.972$, with the normalized eutectic temperature as $T_{E}=1.0$. The images from the simulations are tiled (2x2) times for better visualization. Blue: $\mathrm{Al}_{2} \mathrm{Cu}$, green: $\mathrm{Ag}_{2} \mathrm{Al}$, red: $A l$.

\subsection{Additional microstructures from present study}

In this section, we compute and present additional predictions of possible three-phase co-ordination that could also exist in the selected material systems, by perturbing the original Ag-Al-Cu system that was used in Ref. [21]. Here, for conciseness, we name the $F C C$-phase as $\alpha$, the $H C P$-phase as $\beta$ and the $\theta$-phase as $\gamma$. As a first perturbation, we modified the original parameter set in [21] by switching between the interfacial properties of the $\alpha$ and the $\gamma$-phases. This modification increases the stiffness of the $\gamma$-phase with respect to the $\alpha$-phase leading to the formation of a well connected microstructure for the $\alpha$-phase in which we have rods of the $\beta$-phase, while the originally well connected $\gamma$-phase breaks down, into smaller lamella fragments as displayed in Fig. 9(a). The structure bears similarity to that of a binary eutectic where a rod phase forms in a matrix of the other phase, the distinction here being that we have rod/lamellae fragments of two separate phases of differing volume fractions dispersed in a matrix of the third. 
In the results of the first set of simulations shown in Figs. 8(a) and 8(b), we achieved the required volume fractions (matching the experiments), by perturbing the composition of the $F C C$-phase from the actual values in the CALPHAD databases. As a second alteration, we achieve the same modified volume fractions, but this time by perturbing the liquid compositions, while assuming the same equilibrium composition for the solid-phases as in the CALPHAD database calculations. With this change, the Gibbs-Thomson coefficients of the phases assume different values compared to the previous dataset, making the $\alpha$ phase have the maximum Gibbs-Thomson coefficient. Fig. 9(b) highlights the microstructure that is simulated in this system. In contrast to the earlier simulations where we have the $\alpha$ and $\beta$-phases forming the super-lamellar structure, we now have large rods of the $\alpha$-phase, mixed-states of the $\alpha$ and $\beta$-phases in the matrix of the $\gamma$-phase.

In the third alteration, starting from the dataset in the previous simulation, we modified the stiffness of the $\alpha$-phase by making it the least stiff among the three solid phases. This is performed by changing the solid-liquid surface energy of the $\alpha-l$ interface to a value of 0.32 from its previous value of 0.64 , while the surface energy of the $\beta$-phase is changed to 0.56 from its previous value of 0.62 . Fig. 9(c) displays the microstructure obtained. As expected, the morphology of the $\alpha$-phase changes from a rod-like morphology to a lamellar type, while the other two phases, have rods and lamella fragments of different sizes.

Our final change, was motivated by the morphology in the preceding simulation in Fig. 9(c), where we have lamellar morphology for the $\alpha$-phase (in red), while the $\gamma$-phase (blue) has a fragmented lamellar morphology, interspersed with the $\beta$-phase. The morphology is very similar to the morphology that we derived for the original Ag-Al-Cu data-set [21], where the behavior of the $\alpha$ and $\gamma$-phase is switched, the only difference being, the higher connectivity of the $\gamma$-phase. To change this behavior only slightly to get the super-lamellar connected structure between the $\beta$ and the $\gamma$-phases, we increased the equilibrium volume fractions of the $\beta$-phase only slightly from 0.3 to 0.32. The simulation result is presented in Fig. $9(\mathrm{~d})$. The above change drives the $\beta$-phase morphology towards a more lamellar one, the overall microstructure changes towards the structure of a $\alpha$-phase with a lamellar/lamellarfragment morphology, and a super-lamellar connected state of the $\beta$ and $\gamma$-phases, where the roles of the $\alpha$ and $\gamma$ phases are switched from the original dataset Fig. 8(b), [21]. To put things in perspective, we list in Table 1 the Gibbs-Thomson coefficients for the different simulations performed.

Note that the simulations in Figs. 8(a) and 8(b) can be compared with Fig. 9(a), which differ only in the interfacial properties. Similarly, simulations in Fig. 9(b) and Fig. 9 (c) can be compared with respect to the change in interfacial properties of $\alpha$ (red) and $\beta$ (green) (the minor change in $\gamma-l$ interface is not significant. It was due to a small

\begin{tabular}{|l|l|l|l|}
\hline Simulation & $\alpha$ (red) & $\beta$ (green) & $\gamma$ (blue) \\
\hline $\begin{array}{l}\text { Figs.8(a), } \\
8(\mathrm{~b})\end{array}$ & 0.9371955 & 0.8721219 & 1.4621237 \\
\hline Fig.9(a) & 0.6627729 & 0.8721219 & 1.776791 \\
\hline Fig.9(b) & 1.95137 & 1.047337 & 1.066239 \\
\hline Fig.9(c) & 0.9756848 & 0.9459815 & 1.07519 \\
\hline Fig.9(d) & 0.8930497 & 0.9793346 & 1.009720 \\
\hline
\end{tabular}

Table 1: Gibbs-Thomson coefficients for simulations shown in Figures 8 and 9

difference in the third decimal in the surface energies). The final simulation shows that a difference in the phase diagram as well as the interfacial properties, brings about a greater co-ordination in the ladder structure formed by the $\beta$ (green) and the $\gamma$ (blue) phases which is actually closer to the real experimental results, i.e we have been able to switch the behavior of the phases $\alpha$ and $\gamma$ from the previous results in Figs. 8(a) and 8(b).

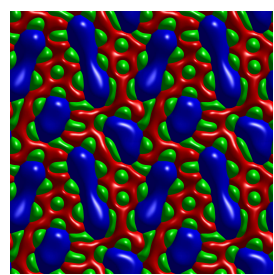

(a)

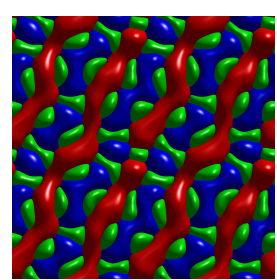

(c)

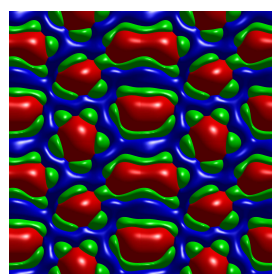

(b)

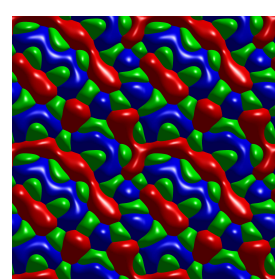

(d)
Figure 9: Hypothetical perturbation of the Ag-Al-Cu system (see text for details). The images are tiled $(2 \times 2)$ for better visualization

\section{Analysis of the morphological features}

In this section, we will briefly show the possibility to compute the variation of certain microstructural features as a function of the processing condition (e.g., variation of composition in the simulations presented in Figs. 4 and 5). In the following, we will parameterize the continuous simulation in Path I by plotting the microstructural features as a function of the volume fraction of the blue phase $A_{\gamma}$. Similarly, simulations along Path II are characterized using the difference of the volume fractions the blue and the red phases $A_{\gamma}-A_{\alpha}$.

An useful measure of the morphological features can be encapsulated as a shape factor of a given phase written as $\Pi=\frac{l_{i}}{\sqrt{A_{i}}}$, where $l_{i}$ is the length of the triple-line and 
$A_{i}$ is the solid-liquid interfacial area associated with the phase $i$. As a comparison, the value for a hemispherical cap surface of a rod morphology in a matrix can be derived as $\approx \frac{\sqrt{2 \pi} \sin (\theta)}{\sqrt{1-\cos (\theta)}}$, where $\theta$ is angle that the solid-liquid interface makes with the horizontal. Similarly, for the projected area of a regular hexagonal configuration, the value for each hexagon is 3.72. Therefore, given a shape-factor for a microstructure, the square of that factor $\Pi^{2}$ can be divided by the representative value of a specific morphology (rod, hexagon, etc.) to derive the number of such phase clusters, which can also be seen as a measure of the scale of the particular phase. Additionally, a plot of the value for different microstructures will allow for a comparison between the morphology of the phase domains.

Fig. 10 highlights the change in the value of the parameter $\Pi$ in the simulations during the composition variations along the different pathways as depicted in Fig. 4 and Fig. 5.

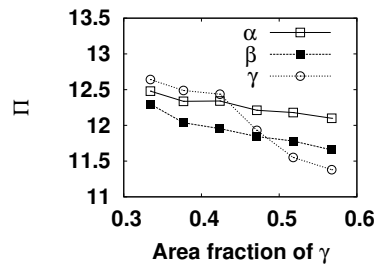

(a)

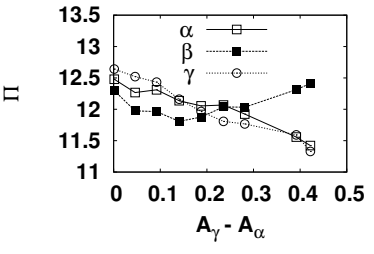

(b)
Figure 10: A shape-factor parameter constructed out of the tripleline length $l_{i}$ and solid-liquid interface area $A_{i}$ of each phase $i$ as $\frac{l_{i}}{\sqrt{A_{i}}}$ is plotted for the two sets of simulations along composition (Path I) in (a) and composition (Path II) in (b) as a function of the volume fractions of the phases. Here $\alpha$ is the red-phase, $\beta$ is the green phase and $\gamma$ is the blue phase.

At the ternary eutectic composition the value of the shape-factor is approximately equal for all the three-phases. With change in volume fractions the value of the shapefactor goes to lower values for both smaller and higher volume fractions of the phases. However, the absolute change in this parameter is small for the entire range of compositions in both simulation conditions along the different composition pathways.

Some other morphological features which can be captured from the simulations are the variation of the fraction of triple-line-length (i.e. the entire length of triple-line belonging to a given phase) as well as the ratio of the linelength of a given phase to its solid-liquid interfacial area fraction. For example, the line-fraction for the case of the $\alpha$ phase, would be $\frac{\alpha \beta+\alpha \gamma}{\alpha \beta+\alpha \gamma+\beta \gamma}$. These measures are plotted as functions of the interface area fraction (also the volume fraction) of the different phases and displayed in Fig. 11.

The plots depict that although the overall line fraction corresponding to each phase is proportional to the vari-

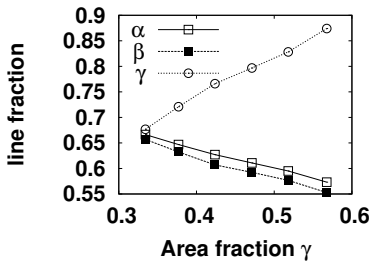

(a)

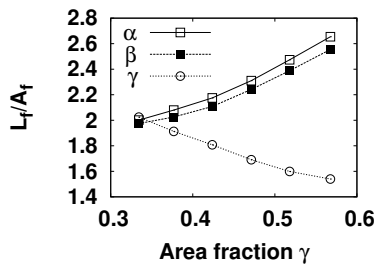

(c)

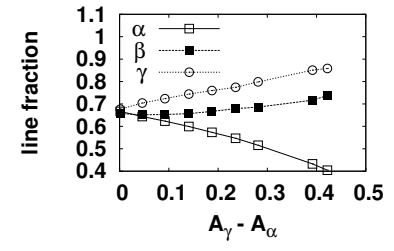

(b)

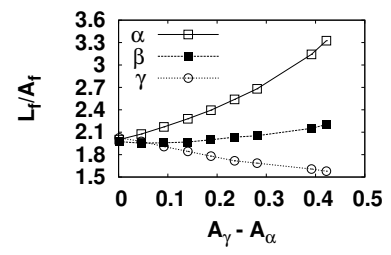

(d)
Figure 11: Plots of the line-fraction as well as the ratio of the linefraction and the interface-fraction for the different simulations along the pathways in (Path I (a) and (c)) and Path II ((b) and (d). In Figs. ((c) and (d)) we introduce $L_{f}$ and $A_{f}$ as short forms for line fraction and area fraction respectively.).

ation of the volume fraction, the ratio of line fraction to the interface area fraction is larger for smaller volume fractions. Also, both the line fraction as well as its ratio with interface area fraction are well correlated with the change in the volume fractions of the phases. The variations observed in the case of the $\beta$ phase (green) for the case of the composition variations along Path II, are minor, and occur as a result of small variation in volume fraction during microstructural transitions.

Lastly, a measure of the asymmetry of the interface shapes can be ascertained by plotting the fraction of the individual triple-line segments with respect to the total triple-line length as a function of the product of the areafractions of the phases forming the interface. The curves displayed in Fig. 12, highlight that, starting from approxi-

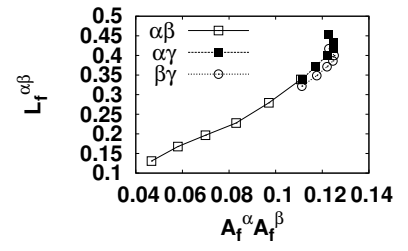

(a)

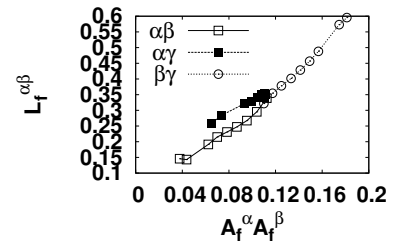

(b)
Figure 12: Plots of the fraction of the length of each triple-line w.r.t to the total triple-line length for the different simulations along the different pathways in (Path I (a)) and Path II ((b). $L_{f}^{\alpha \beta}$ represents the line fraction of the given triple-line, while $\mathrm{A}_{f}^{\alpha} \mathrm{A}_{f}^{\beta}$ represents the product of the respective solid phase-fractions forming a given tripleline).

mately equal line fractions of each interface at the ternary 
eutectic composition, for both composition pathways, the $\alpha-\beta$ (red-green) line fraction is the least, following the product of the volume fractions of the $\alpha$ (red) and the $\beta$ (green) phases which decreases with change in volume fractions of the phases. While for the composition pathway (Path I, Fig. 12(a)), the interface fractions of the $\alpha-\gamma$ (red-blue) and $\beta-\gamma$ (green-blue) interfaces increase with time. For the case of composition pathway II (Fig. $12(\mathrm{~b}))$, both $\alpha-\gamma$ (red-blue), $\alpha-\beta$ (red-green) interfaces reduce in fraction, all of them correlating very well with the variation of the product of the volume fractions of the concerned solid-phases forming a given triple-line with the liquid. Additionally, for the case of Path II (Fig. 12(b)), the curve for the $\alpha-\gamma$ interface seems to deviate from that of the line-fraction variations of the $\alpha-\beta$ and $\beta-\gamma$ variations as a function of the product of the volume fractions. Also note, both sets of simulations represented by points on Fig. 12(a) and Fig. 12(b) show certain abrupt changes, which correspond to points of morphological transitions. Therefore, there seems to be a weak correlation of the variation of individual line-fractions, with the shapefactor.

Transitions to different morphologies occur through loss of $\alpha-\beta$ interfaces in both sets of simulations(Path I and Path II) causing decrease in the number of $\alpha-\beta$ segments and thereby increase in the length of individual $\alpha-\beta$ segments for the same given line fraction of the $\alpha-\beta$ interface. Along both composition pathways, complete breakdown of the hexagonal configuration occurs for a triple-line fraction corresponding to the $\alpha-\beta$ interface around 0.15 .

So, in summary for a continuous set of controlled simulations such as those performed along the composition pathways one can indeed track the variation of certain morphological features. This also helps in deriving correlations/heuristics for the variation of some of the microstructural features and configurations in response to the imposed conditions. However, for a generic simulation it is difficult to derive what is the parameter set of microstructural measures that is going to be characteristic of a given collection of microstructures. So for example, in the previous simulations there is also the variation of the mean number of nearest neighbors which evolves during the configuration changes. Similarly, there are others that are not considered such as the correlations between the arrangement of the different rods with respect to themselves as well as the other phases. In the following section, we therefore adopt a statistical method of representing microstructures which will allow us to compare between the entire set of microstructures that were produced using microstructure descriptors that could even be composite functions of several individual microstructural features.

\section{Data-driven classification of microstructures}

Most of the methods used in microstructure quantification include only the very basic descriptors such as volume fraction, average grain size, average number of nearest neighbors, etc. Such basic measures can be inadequate or even be potentially misleading in extracting reliable process-structure-property linkages [22-28]. For example, it is easy to envision structures with the same values of above-mentioned measures with very different properties. In the present work, we employ the recently developed and demonstrated framework [22, 23, 29-31] for microstructure quantification based on two-point statistics and principal component analysis (PCA). Details of this new framework are summarized next.

\subsection{Framework for microstructure quantification}

Two-point statistics (i.e., 2-point spatial correlations) are the most basic of the n-point spatial correlations that can be used to capture the details of the microstructure $[22,23,29-37]$. These can be defined as

$$
f_{r}^{h h^{\prime}}=\frac{1}{S} \sum_{s=1}^{S} m_{s}^{h} m_{s+r}^{h^{\prime}}
$$

where $m_{s}^{h}$ is the digital representation of microstructure denoting the volume fraction of local state labelled $h$ present in the spatial cell or voxel labelled $s . f_{r}^{h h^{\prime}}$ then denotes the joint probability of finding local states $h$ and $h^{\prime}$ at spatial locations separated by a vector $r$ in the entire microstructure. Implicit in these expressions is the use of a consistent discretization and enumeration scheme for both the microstructure volume and the vector space (corresponding to $r$ in Eq. 1).

The calculation of 2-point statistics defined in Eq. 1 can be performed in a computationally efficient manner by employing discrete Fourier transforms (DFT) [22, 23, 2933 . However, the computation produces a very large set of descriptors for the material structure. Indeed, the number of descriptors is equal to the number of distinct discretized vectors included in the analyses. For example, if one were to define a $21 \times 21$ neighborhood (which corresponds to including only ten voxels on each side of the voxel of interest) in a 2-D microstructure (or a cross-section of a 3D microstructure), one ends up with 441 descriptors of the material structure. Clearly, this number of material structure descriptors will be unwieldy for any subsequent analyses (e.g., extraction of process-structure-property linkages). In prior work, [22, 23, 29-31], principal component analysis (PCA) has been demonstrated to provide objective low dimensional representation of the 2-point statistics of the material microstructure. PCA essentially projects high dimensional data onto a new rotated orthogonal coordinate system whose directions are ordered from highest to lowest variance in the given dataset. In other words, this approach represents a data-driven (objective) approach for low-dimensional representation of high dimensional data.

Let $r=1,2, \ldots, R$ denote the number of dimensions in the set of 2-point statistics, $f_{r}$, deemed to be of interest in quantifying a given microstructure. Let $k=1,2, \ldots, K$ enumerate each element in the ensemble of microstructures being studied. Assuming that $R$ is typically much 
larger than $K$, PCA defines a new coordinate system with a maximum of $K-1$ orthogonal directions, which are in a descending order of the variance. This implies that 2point statistics of a realization can be represented within machine precision by using $K-1$ parameters. Two-point statistics of a realization indexed by $(k)$ can now be expressed in the space of principal components as

$$
f_{r}^{(k)}=\sum_{i=1}^{\min ((K-1), R)} \alpha_{i}^{(k)} \varphi_{i r}+\bar{f}_{r}
$$

where $\varphi_{\text {ir }}$ are the components of the orthogonal set of directions specified by PCA, $\alpha_{i}^{(k)}$ are PC weights of $k^{t h}$ element of the ensemble, and $\bar{f}_{r}$ represents the ensemble averaged 2-point statistics. The inherent variance in the entire ensemble of microstructures is captured in the eigenvalues, $b_{i}$, computed as a part of PCA. Eigenvalues convey the importance of each principal component. By keeping only the principal components with the highest eigenvalues, an objective reduced-order representation of the microstructure can be obtained with a relatively small number of terms (compared to the dimensionality of the complete set of 2-point statistics). Mathematically, this reduced-order representation can be expressed as

$$
f_{r}^{(k)} \approx \sum_{i=1}^{R^{*}} \alpha_{i}^{(k)} \varphi_{i r}+\bar{f}_{r}
$$

where $R^{*}$ is typically very small compared to $\min ((K-$ $1), R)$. If one adopts this approach, $\alpha_{i}^{(k)}, i=1,2, \ldots, R^{*}$ denote the salient measures of a selected microstructure $(k)$. These are also referred as PC scores or PC weights, and are often simply denoted as $P C i, i=1,2, \ldots, R^{*}$.

\subsection{Application to microstructures}

The total number of spatial correlations that can be defined in a three phase material system is nine. This can be deduced by listing all permutations of $h$ and $h^{\prime}$. Three of these correlations are autocorrelations where $h=h^{\prime}$ and six of them are crosscorrelations where $h \neq h^{\prime}$. In prior work [38], it was shown that for a material system with $H$ number of distinct phases, only $H-1$ correlations are independent. In this study, we used autocorrelation of the blue colored phase and its crosscorrelation with the red colored phase in the reduced order representation of the microstructure due to the fact that blue phase and red phase exhibit the first and second largest ranges of volume fraction values (i.e., one-point statistics of the microstructures), respectively. Thus, the statistical representation of microstructure for this study yields a $2 S$ dimensional space, where $S$ is the total number of spatial bins used to describe the microstructure.

As an example, Figure 13 shows the autocorrelation and crosscorrelation for the microstructure shown in Figure $4(\mathrm{a}))$. The statistical distributions are truncated to a vector size of 500 voxels in both $\mathrm{x}$ and $\mathrm{y}$ directions. The

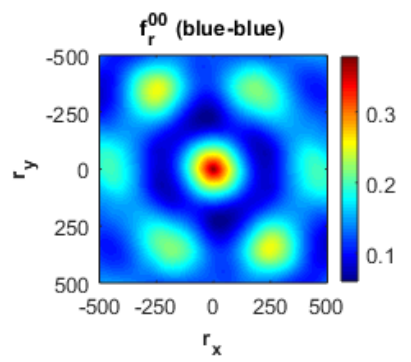

(a)

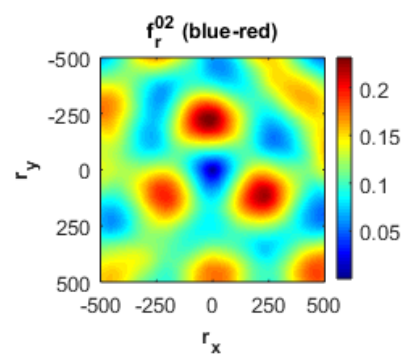

(b)
Figure 13: Quantification of the microstructure in Figure 4(a). (a) Autocorrelation of blue phase, and (b) the crosscorrelation between blue and red phases.

original microstructure has $S=1694^{2}=2869636$ number of pixels. The truncation of the vector space described above still leads to $1001^{2}=1002001$ vectors. This truncation is usually justified by the fact that the microstructure is generally uncorrelated for large vectors (i.e., for large vectors the joint probability defined by 2-point statistics is merely the product of the individual 1-point statistics involved).

Note that the correlations shown in Figure 13 contain tremendous amount of information regarding the material microstructure. The center value (corresponding to $r=0$ vector) in the autocorrelation provides the volume fraction of phase $h$, while the corresponding value in the crosscorrelation is zero as we do not allow the different phases to co-exist in any single spatial cell or voxel [33]. Each peak (valley) in the correlations points to abundance (scarcity) of examples of points with specified local states separated by a specified vector (starts at the center of the correlation plot and ends at the peak/valley location). Note also that these correlations carry information on the anisotropy of the material structure.

The large ensemble of simulation results presented earlier (with different compositions and surface energies; Figures $4,5,6,7,8,9)$ ) and the experimental micrograph in Figure 1(a) were analyzed using the protocols described earlier. PCA of the 2-point statistics provides a useful lowdimensional representation and visualization of the entire ensemble of microstructures. The bottom left plot in Figure 14 is called the scree plot and provides information on the contribution of each PC in capturing the overall variance in the entire dataset. For example, for the ensemble of microstructures studied, it is seen that the first 3 PCs account for $90 \%$ of the total variance in the entire dataset. The top left plot shows the representations of the microstructures studied in the first three PCs. In order to help visualize this 3-D plot better, we have also shown the 2 -D projections of the same plot in the right column of the same figure. Note that the microstructures from each earlier figure in the paper are represented by the same color and are labeled following the exact same notation (as was used in the earlier figures in this paper). As an example, the effect of composition can be seen clearly as trendlines 

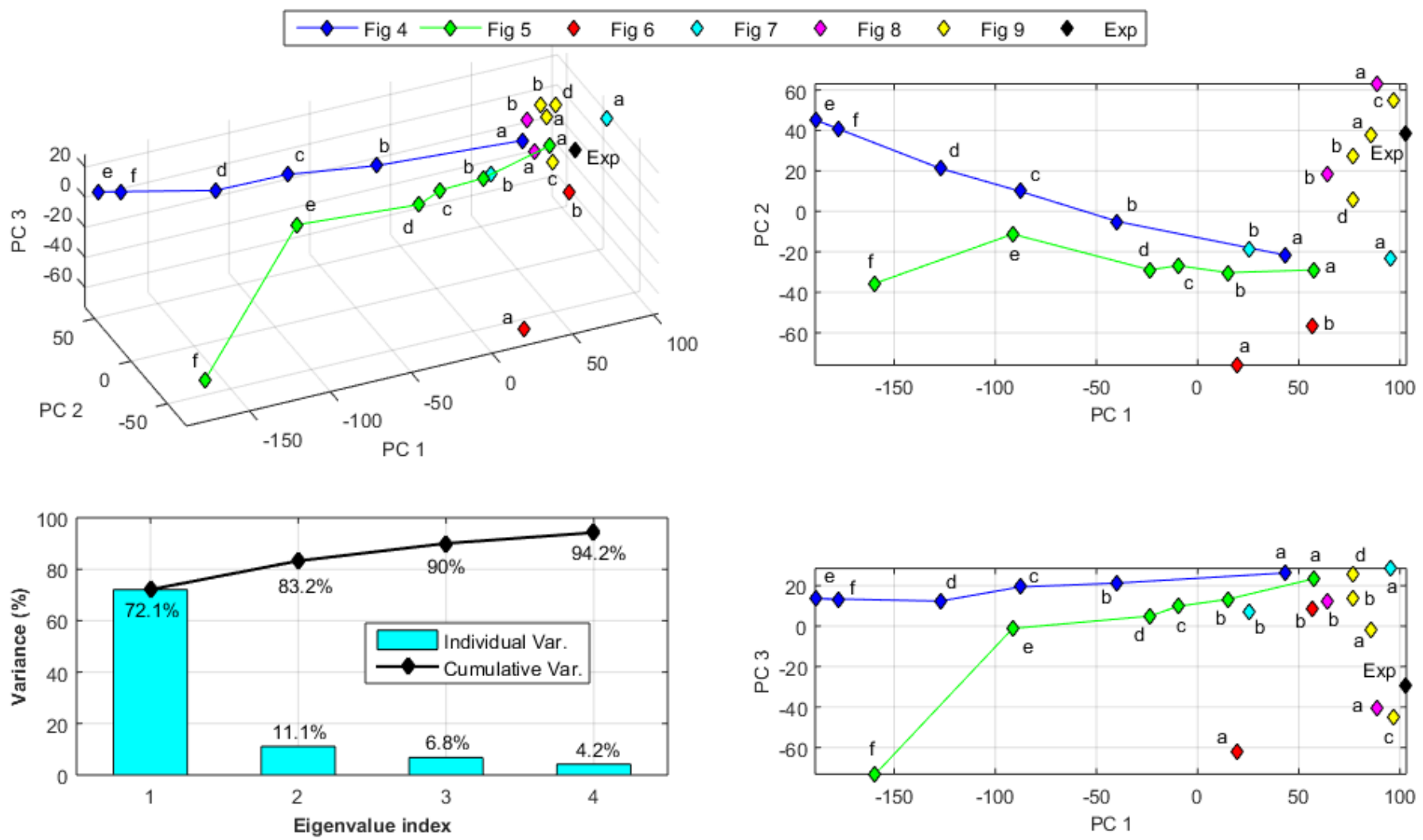

Figure 14: PC scores of microstructures obtained with different compositions and surface energies. The top left plot depicts the microstructures in the reduced-order 3-D PC space. The top and bottom right plots show 2-D projections of the top left 3-D plot to enable closer inspection. Bottom left plot shows the individual and cumulative variances from the PCA for the first 4 principal components

in the set of microstructures labelled $4 \mathrm{a}$ to $4 \mathrm{f}$ (identified as Path I) and in the set of microstructures labelled 5a to $5 f$ (identified as Path II).

In Figure 15, the first 3 basis vectors for both the autocorrelation and crosscorrelation can be seen. In the top row, there are 3 basis vectors for autocorrelation which correspond to the $3 \mathrm{PCs}$ with highest variance and in the bottom are the corresponding basis vectors for the crosscorrelation. The peaks and valleys in each basis plot identify the dominant 2-point statistics captured in each basis. In other words, as PC1 is increased, the set of 2-point statistics that experience the biggest change are the ones identified as peaks and valleys in the first basis plots (for both autocorrelations and crosscorrelations) (cf. Eq. 3). Therefore, a careful study of the basis plots with the PC scores has the potential to provide new quantitative insights on how compositions and surface energies affect the material microstructure. Data science tools for these analyses are not yet fully developed.

The microstructures in Figures 4(a) and 5(a) are very similar to each other; indeed they are also very close to each other in the low-dimensional PC representations shown in Figure 14. A consistent observation from the numerous prior applications of the methods employed here is that PC1 generally shows a strong correlation with the volume
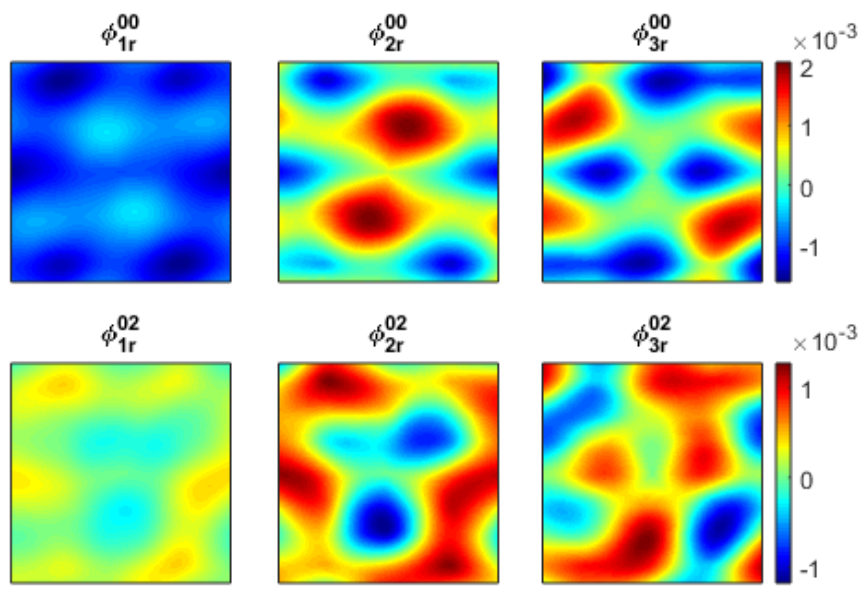

Figure 15: Basis vectors for first 3 principal components obtained in the PCA employed in this work. Top row shows the first 3 basis vectors for blue phase - blue phase autocorrelation and bottom row shows the corresponding basis vectors for blue phase - red phase crosscorrelation. For clarity, colorbars are only shown on the rightmost plots; these are the same for each row of plots shown above. Axes values and axes labels are the same as in Figure 13. 
fraction measures of the microstructure. In the present case, it is observed that as the volume fraction of blue phase increases, the PC1 values decreased substantially (see the trendlines shown in Figure 14).

Note further that the trendlines reveal that the microstructures in the two paths depicted are relatively similar up to a certain point (compare 6e and 7c) and then they begin to diverge. This is of course also discernable directly in the micrographs themselves. Note also that microstructures in Figures 5(f) and 6(a) are distinctly different from the others (they exhibit continuous channels of blue phases going from one side to the other side); indeed these are far away from the other microstructures in the $\mathrm{PC}$ representations as well. The fact that the trends in microstructure changes are captured so remarkably well in the highly reduced dimensional representation (only three PCs were used) provides tremendous support to the validity and power of the data-driven approaches for microstructure quantification employed in this work.

Comparison of microstructures in Figures 6(b) and 7(a) helps us visualize the effect of surface energy on the microstructure morphology. Similarly, microstructures in Figure 4(a) and Figure 7(b) can also be compared for the same purpose. In both these comparisons, the compositions are similar, while the surface energies are changed. In the PC representations, this is reflected by the fact that the biggest changes within these sets of microstructures is in the values of PC2 and PC3. These two components appear to control the strength of the hexagonal arrangements in the microstructures. It appears that negative values of $\mathrm{PC} 2$ and positive values of PC3 result in strong hexagonal arrangements for the red phase or the 120 degree pattern between the blue phase and the nearest red phases. These same patterns can also be deduced in the second and third basis plots of the blue phase - red phase crosscorrelations in Figure 15. It should therefore be clear that the fundamental patterns in the microstructures are indeed efficiently captured by the PCA. The most beneficial aspect of PCA is that the ensemble is viewed from the most succinct viewpoint in terms of capturing the variance in the ensemble. However, this does come at a price that we are yet unable to extract a precise physical meaning of the PCs. Coupling the statistical analysis described above with some more controlled simulations could lead to greater understanding. For example, Fig.16 highlights the influence of the change in surface energy, wherein a smaller solid-liquid interfacial energy leads to elongation of the particular phase. This can be attributed to the change in the characteristics of the quadruple points which also correlates well with the extended simulations previously performed in Fig.7(a).

Similarly, smaller simulations along the composition pathways also reveal the tendency to connect at larger volume fractions as highlighted in Fig.17, which compares well with the extended simulations in Figs. 4 and 5 except that the nature of connectivity is different in the two extended simulations, which is captured in the statistical analysis.

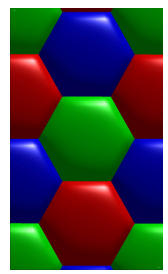

(a)

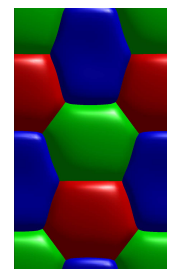

(b)
Figure 16: Simulations in smaller simulation boxes respecting the geometry of the hexagon. In (a) all surface energies are equal and we get regular hexagons, whereas in (b) the solid-liquid interfacial energy of the blue phase is smaller than the others leading to elongation of the phase along a prescribed direction.

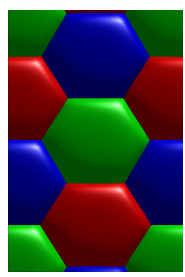

(a)

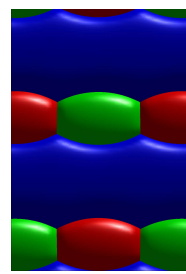

(b)
Figure 17: Simulations in smaller simulation boxes respecting the geometry of the hexagon. In (a) the volume fractions are close to the symmetric ternary eutectic point while in (b) they correspond to a volume fraction well into Path I of the composition pathway in Fig.2.

In fact one can repeat the continuous composition simulation along Path I for lower solid-liquid interfacial energies of one of the phases. The end-state connectivity is very different from the one derived with equal surface energies as seen in Fig.18. This can be related to the biased elongation of the blue phase due to lower surface energy as seen in Fig.16.

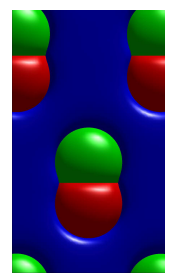

Figure 18: Continuous simulation along Path I with lower surface energy of the blue-phase leads to a different connectivity of the blue phase with increase in volume fraction than in the case of equal surface energies.

One of the benefits of PCA is that a distance between two microstructures, $D$, can be reliably calculated as

$$
D=\sqrt{\sum_{i=1}^{R^{*}}\left(\alpha_{i}^{(1)}-\alpha_{i}^{(2)}\right)^{2}}
$$

where $\alpha_{i}^{(1)}$ and $\alpha_{i}^{(2)}$ are $i$ th PC weigths of first and sec- 
ond microstructures, respectively. $R^{*}$ is the total number of principal components that are retained in the reduced order representation of microstructure. The main reason this distance measure is quite reliable comes from the fact that the transformation involved in the PCA is a distance preserving transformation (i.e., a rotational transformation).

Using Eq. 4, we can quantitatively compare the experimental micrograph in Figure 1(a) with the simulated ones. It was found that the simulated microstructure in Figure 8(a) is closest to the experimental micrograph after a suitable rotation of the experimental micrograph (i.e., to match the orientations of chains containing red and green phases).

\begin{tabular}{|l|c|}
\cline { 2 - 2 } \multicolumn{1}{c|}{} & Exp \\
\hline Fig 4(a) & 105.6137 \\
\hline Fig 4(b) & 161.3428 \\
\hline Fig 4(c) & 202.7718 \\
\hline Fig 4(d) & 237.0239 \\
\hline Fig 4(e) & 297.0911 \\
\hline Fig 4(f) & 285.6307 \\
\hline Fig 5(a) & 100.2349 \\
\hline Fig 5(b) & 121.7070 \\
\hline Fig 5(c) & 138.4646 \\
\hline Fig 5(d) & 151.1770 \\
\hline Fig 5(e) & 206.5472 \\
\hline Fig 5(f) & 282.3201 \\
\hline
\end{tabular}

\begin{tabular}{|l|c|}
\cline { 2 - 2 } \multicolumn{1}{c|}{} & Exp \\
\hline Fig 6(a) & 156.2755 \\
\hline Fig 6(b) & 119.9401 \\
\hline Fig 7(a) & 98.8067 \\
\hline Fig 7(b) & 116.2587 \\
Fig 8(a) & 35.9418 \\
\hline Fig 8(b) & 87.6246 \\
\hline Fig 9(a) & 71.9712 \\
\hline Fig 9(b) & 96.3519 \\
\hline Fig 9(c) & 53.9416 \\
\hline Fig 9(d) & 101.4887 \\
\hline
\end{tabular}

Table 2: Euclidean distances between experimental micrograph shown in Figure 1(a) and each microstructure shown in Figures 4, 5, $6,7,8,9$. The distances are calculated according to Eq. 4 by using the first 10 principal components.

\section{Conclusion}

In this paper, we have have highlighted the influence of the volume fractions and surface energies on three-phase growth patterns using both phase-field simulations and numerical characterization of the microstructures. The statistical analysis allows to quantify the differences in the obtained microstructures. The key result of the analysis suggests that while it is still no clear way to predict what patterns will be selected given a set of conditions, however some clear indications of the influence of the change of volume fractions (alloy composition) and surface energies from a given set of parameter and processing conditions can be predicted. Here both, lowering the solid-liquid interfacial energies of a given phase or increase in volume fractions lead to increase in connectivity of the phases. However, the topological mechanisms through which connectivity among the phases is enhanced, is different between the cases of increase in volume fractions and decrease in solid-liquid interfacial energy. It was demonstrated that the data-driven protocols are very efficient in providing a concise and efficient classification of the microstructures based on their intrinsic morphologies. These can in turn motivate or guide additional controlled simulations that can lend a greater understanding about the role of the different parameters in pattern formation.

\section{Acknowledgments}

A.C would like to thank Mathis Plapp, Lorenz Ratke, Britta Nestler for giving their impressions and thoughts about the manuscript as well as for the valuable insights in the work. YCY and SRK acknowledge support from AFOSR award FA9550-12-1-0458. AD acknowledges support by Deutsche Forschungsgemeinschaft (DFG; project No. RA537/14-2).

\section{References}

[1] S. Rex, B. Böttger, V. T. Witusiewicz, U. Hecht, Transient eutectic solidification in In-Bi-Sn: Two-dimensional experiments and numerical simulation, Materials Science and Engineering A 413 (2005) 249-254.

[2] V. T. Witusiewicz, U. Hecht, L. Sturz, S. Rex, Phase equilibria and eutectic growth in quaternary organic alloys amino-methylpropanediol-(D)camphor-neopentylglycol-succinonitrile (AMPD-DC-NPG-SCN), J. Cryst. Growth 297 (2006) 117.

[3] H. W. Kerr, A. Plumtree, W. C. Winegard, The structure of the tin-lead-cadmium eutectic, Journal of the Institute of Metals 93 (1964) 63.

[4] H. A. Q. Bao, F. C. L. Durand, Morphologie eutectique dans le système Cd-Pb-Sn, J. Cryst. Growth 15 (1972) 291.

[5] D. J. S. Cooksey, A. Hellawell, The microstructures of ternary eutectic alloys in the systems Cd-Sn-(Pb-In-Tl), Al-Cu(Mg, Zn, Ag), Zn-Sn-Pb, Journal of the Institute of Metals 95 (1967) 183.

[6] J. D. Holder, B. F. Oliver, The directional solidification of PbSn-Cd alloys, Metallurgical Transactions 5 (1974) 2423.

[7] M. D. Rinaldi, R. M. Sharp, M. C. Flemings, Growth of ternary composites from the melt, Metallurgical Transactions 3 (1972) 3139 .

[8] M. A. Ruggiero, J. W. Rutter, Origin of microstructure in 350 $\mathrm{K}$ eutectic of Bi-In-Sn ternary system, Materials Science and Technology 11 (1995) 136.

[9] D. G. McCartney, J. D. Hunt, R. M. Jordan, The structures expected in a simple ternary eutectic system: Part 1. Theory, Metallurgical Transactions A 11A (1980) 1243.

[10] T. Himemiya, T. Umeda, Three-phase planar eutectic growth models for a ternary eutectic system, Materials Transactions JIM 40 (7) (1999) 665-674.

[11] M. Apel, B. Boettger, V. Witusiewicz, U. Hecht, I. Steinbach, Solidification and Crystallization, Wiley-VCH, 2004.

[12] A. Choudhury, M. Plapp, B. Nestler, Theoretical and numerical study of three-phase growth in ternary alloys, Phys. Rev. E 83 (2011) 051608.

[13] D. G. McCartney, R. M. Jordan, J. D. Hunt, The structures expected in a simple ternary eutectic system: Part II. The AlAg-Cu ternary system, Metallurgical Transactions A 11 (1980) 1251.

[14] U. Hecht, L. Gránásy, T. P. B. Böttger, M. Apel, V. Witusiewicz, L. Ratke, J. D. Wilde, L. Froyen, D. Camel, B. Drevet, G. Faivre, S. G. Fries, B. Legendre, S. Rex, Multiphase solidification in multicomponent alloys, Mat. Sci. Eng. R 46 (2004) 1.

[15] A. Genau, L. Ratke, Crystal orientation and morphology in AlAg-Cu ternary eutectic, IOP Conf. Series: Mat. Sci and Engg. 27 (2011) 012032.

[16] A. Genau, L. Ratke, Morphological characterization of the $\mathrm{Al}-\mathrm{Ag}-\mathrm{Cu}$ ternary eutectic, International Journal of Materials Research 103 (2012) 469. 
17] A. Dennstedt, L. Ratke, Microstructures of Directionally Solidified $\mathrm{Al}-\mathrm{Ag}-\mathrm{Cu}$ Ternary Eutectics, Transactions of the Indian Institute of Metals 65 (2012) 777-782.

[18] A. Dennstedt, L. Ratke, New Metallographic Method for Estimation of Ordering and Lattice Parameter in Ternary Eutectic Systems, Metallogr. Microstruct. Anal. 2 (2013) 140-147.

19] R. J. Contieri, C. T. Rios, M. Zanotello, R. Caram, Growth and three-dimensional analysis of a $\mathrm{Nb}-\mathrm{Al}-\mathrm{Ni}$ ternary eutectic, Materials Characterization 59 (2008) 693.

[20] A. Dennstedt, A. Choudhury, L. Ratke, B. Nestler, Microstructures in a ternary eutectic alloy, submitted to IOP Conf. Series - Materials Science and Engineering.

[21] A. Choudhury, Pattern formation during self-organization in three-phase eutectic solidification, Transactions of the Indian Institute of Metals 68 (2015) 1137-1143.

22] A. Gupta, A. Cecen, S. Goyal, A. K. Singh, S. R. Kalidindi, Structure-property linkages using a data science approach: Application to a non-metallic inclusion/steel composite system, Acta Materialia 91 (2015) 239-254.

[23] A. Çeçen, T. Fast, E. Kumbur, S. Kalidindi, A data-driven approach to establishing microstructure--property relationships in porous transport layers of polymer electrolyte fuel cells, Journal of Power Sources 245 (2014) 144-153.

[24] Y. C. Yabansu, D. K. Patel, S. R. Kalidindi, Calibrated localization relationships for elastic response of polycrystalline aggregates, Acta Materialia 81 (2014) 151-160.

[25] Y. C. Yabansu, S. R. Kalidindi, Representation and calibration of elastic localization kernels for a broad class of cubic polycrystals, Acta Materialia 94 (2015) 26-35.

[26] R. Liu, Y. C. Yabansu, A. Agrawal, S. R. Kalidindi, A. N. Choudhary, Machine learning approaches for elastic localization linkages in high-contrast composite materials, Integrating Materials and Manufacturing Innovation 4 (1) (2015) 13.

[27] G. Landi, S. R. Niezgoda, S. R. Kalidindi, Multi-scale modeling of elastic response of three-dimensional voxel-based microstructure datasets using novel DFT-based knowledge systems, Acta Materialia 58 (7) (2010) 2716-2725.

[28] T. Fast, S. R. Kalidindi, Formulation and calibration of higherorder elastic localization relationships using the MKS approach, Acta Materialia 59 (11) (2011) 4595-4605.

[29] S. R. Niezgoda, Y. C. Yabansu, S. R. Kalidindi, Understanding and visualizing microstructure and microstructure variance as a stochastic process, Acta Materialia 59 (16) (2011) 6387-6400.

[30] S. R. Niezgoda, A. K. Kanjarla, S. R. Kalidindi, Novel microstructure quantification framework for databasing, visualization, and analysis of microstructure data, Integrating Materials and Manufacturing Innovation 2 (1) (2013) 1-27.

[31] P. Steinmetz, Y. C. Yabansu, J. Hötzer, M. Jainta, B. Nestler, S. R. Kalidindi, Analytics for microstructure datasets produced by phase-field simulations, Acta Materialia 103 (2016) 192-203.

[32] D. T. Fullwood, S. R. Niezgoda, B. L. Adams, S. R. Kalidindi, Microstructure sensitive design for performance optimization, Progress in Materials Science 55 (6) (2010) 477-562.

[33] D. T. Fullwood, S. R. Niezgoda, S. R. Kalidindi, Microstructure reconstructions from 2-point statistics using phase-recovery algorithms, Acta Materialia 56 (5) (2008) 942-948.

34 ] B. L. Adams, X. C. Gao, S. R. Kalidindi, Finite approximations to the second-order properties closure in single phase polycrystals, Acta Materialia 53 (13) (2005) 3563-3577.

[35] A. W. Moore, A. J. Connolly, C. Genovese, A. Gray, L. Grone, N. Kanidoris II, R. C. Nichol, J. Schneider, A. S. Szalay, I. Szapudi, et al., Fast algorithms and efficient statistics: N-point correlation functions, in: Mining the Sky, Springer, 71-82, 2001.

36] A. G. Gray, A. W. Moore, N-Body'problems in statistical learning, in: NIPS, vol. 4, Citeseer, 521-527, 2000.

[37] A. Cecen, T. Fast, S. R. Kalidindi, Versatile algorithms for the computation of 2-point spatial correlations in quantifying material structure, Integrating Materials and Manufacturing Innovation 5 (1) (2016) 15.

[38] S. Niezgoda, D. Fullwood, S. Kalidindi, Delineation of the space of 2-point correlations in a composite material system, Acta 

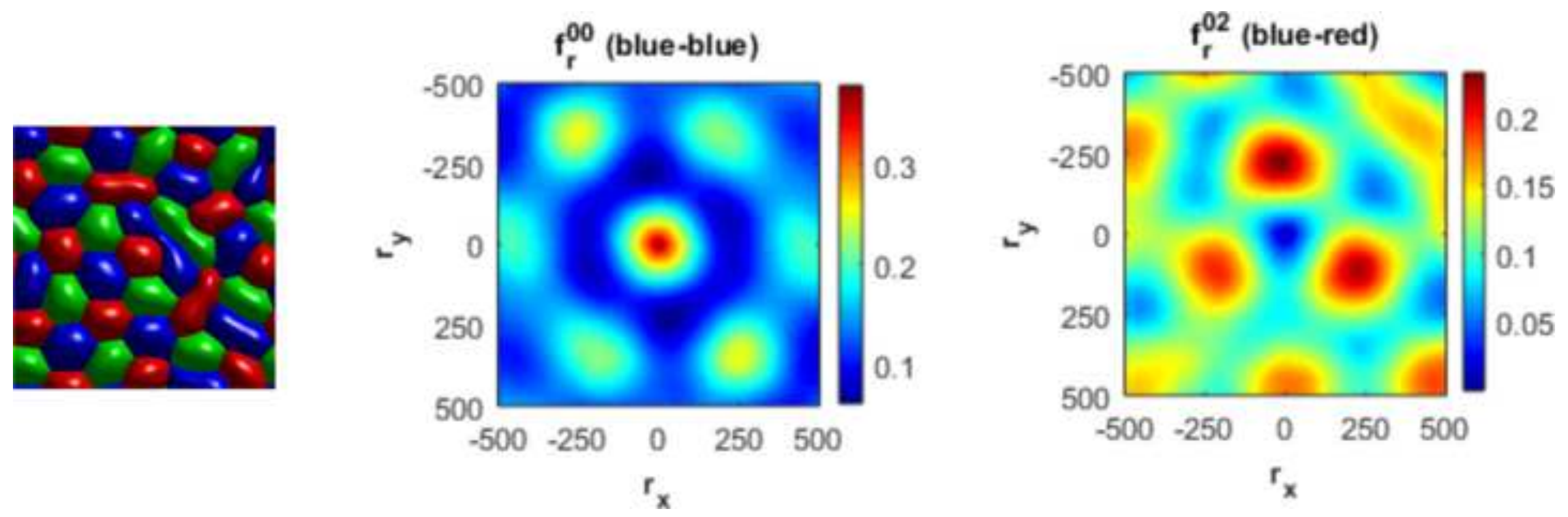